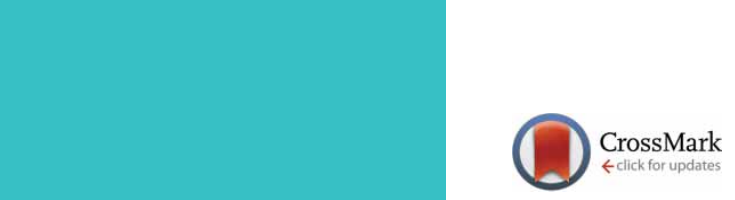

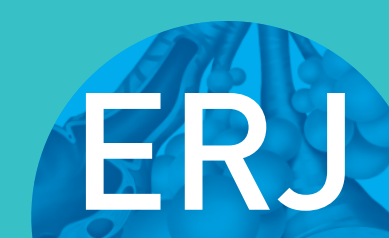

open research
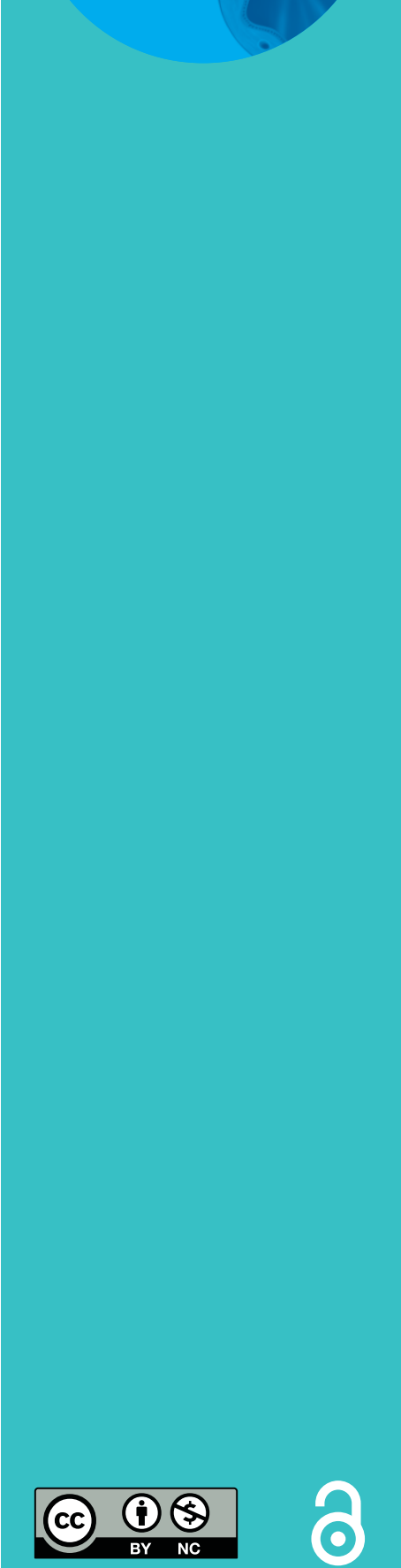

\section{Physical activity for cystic fibrosis: perceptions of people with cystic fibrosis, parents and healthcare professionals}

\author{
Sarah Denford (1) ${ }^{1}$, Narelle S. Cox², Kelly A. Mackintosh ${ }^{3}$, Melitta A. McNarry ${ }^{3}$, \\ Paul O'Halloran4, Anne E. Holland (1) ${ }^{2}$, Owen W. Tomlinson (1) ${ }^{1}$, Alan R. Barker ${ }^{1}$ \\ and Craig A. Williams ${ }^{1}$, on behalf of Youth Activity Unlimited - A Strategic \\ Research Centre of the UK Cystic Fibrosis Trust
}

Affiliations: ${ }^{1}$ Children's Health and Exercise Research Centre, Sport and Health Sciences, University of Exeter, Exeter, UK. ${ }^{2}$ Monash University, School of Primary and Allied Health Care, Frankston, Victoria, Australia. ${ }^{3}$ Applied Sports Science, Technology, Exercise and Medicine Research Centre (A-STEM), Swansea University, Bay Campus, Swansea, UK. ${ }^{4}$ School of Psychology and Public Health, La Trobe University, Bundoora, Victoria, Australia.

Correspondence: S. Denford, Children's Health and Exercise Research Centre, Sport and Health Sciences, University of Exeter, St Luke's Campus, Heavitree Road, Exeter, EX1 2LU, UK. E-mail: S.Denforddexeter.ac.uk

\section{ABSTRACT}

Background: The benefits of physical activity (PA) for people with cystic fibrosis (pwCF) are widely accepted, yet how PA is promoted and utilised by pwCF is unclear.

Method: An online questionnaire to explore attitudes, practices and promotion of PA in cystic fibrosis was completed by healthcare providers (HCP), pwCF and parents/caregivers.

Results: 351 respondents (105 HCP, 120 pwCF, and 126 parents/caregivers) from 12 countries completed the survey. Importance of PA was rated highly by the majority of respondents. Physical (e.g. health), psychological (e.g. enjoyment) and social (e.g. social interaction) factors were motives for PA for $82 \%, 49 \%$ and $37 \%$ of pwCF, respectively, irrespective of country. Common barriers to PA included time (49\% and $36 \%)$ and tiredness $(61 \%$ and $7 \%)$ for pwCF and parents/carers, respectively. pwCF also reported psychosocial barriers (e.g. stigma, demoralisation), while parents/caregivers reported structural barriers (e.g. cost). Clinical teams varied substantially in terms of the emphasis placed on PA, facilities available, staff and training, and advice given to pwCF.

Conclusion: Despite the majority of participants rating the importance of PA highly, substantial variability was evident regarding the facilities and clinical support available to them, as well as why and how people were active. There remains a need to identify what constitutes "best practice" for PA promotion within clinics.

@ERSpublications

Despite rating the importance of physical activity for cystic fibrosis highly, substantial variation is evident in why and how people are active, and facilities and support available https://bit.ly/ $392 \mathrm{i} 8 \mathrm{Cn}$

Cite this article as: Denford S, Cox NS, Mackintosh KA, et al. Physical activity for cystic fibrosis: perceptions of people with cystic fibrosis, parents and healthcare professionals. ERJ Open Res 2020; 6: 00294-2019 [https://doi.org/10.1183/23120541.00294-2019].

This article has supplementary material available from openres.ersjournals.com

Received: 28 Oct 2019 | Accepted after revision: 14 July 2020

Copyright $\odot$ ERS 2020. This article is open access and distributed under the terms of the Creative Commons Attribution Non-Commercial Licence 4.0. 


\section{Introduction}

The physical and psychosocial health benefits of physical activity (PA), including exercise, sport and recreation, are widely recognised $[1,2]$ and recommended in the management of cystic fibrosis $(\mathrm{CF})[3,4]$. Although it has been suggested that individuals with CF are not as active as their peers [5], a more recent, objective PA study suggests that, overall, PA levels in people with cystic fibrosis (pwCF) are similar to those in their age-matched peers [6]. However, the vast majority of youth, with and without CF, are not meeting the recommended 60 min of moderate-to-vigorous PA per day, and this inactivity increases with age [7].

Healthcare providers and patients have both indicated that PA is considered a high priority in the treatment of CF, but many questions remain to be answered, as highlighted in the recent James Lind Alliance Priority Setting Partnership in CF in the UK [8]. However, the views, opinions and practices of pwCF, and their support teams, regarding the promotion of, and engagement in, PA are understudied, limiting the ability to develop effective interventions to promote PA in this population.

Many factors influence whether an individual is physically active, such as age, sex, knowledge, attitudes and/or motivations [9]. The social environment (e.g. active families), and the built environment (e.g. access to facilities within hospitals, parks and green space) in which young people live and interact will also influence PA levels [10]. However, the impact of such factors on PA levels in pwCF is not clear. In previous qualitative studies, pwCF and healthcare professionals (HCP) discussed a range of individual, interpersonal and environmental motives for, and barriers and facilitators to, PA and PA promotion [11]. For pwCF, barriers include a low value assigned to PA [12], unpleasant connotations associated with PA [13] and a lack of opportunity for PA [13]. HCP reported a lack of clarity regarding how PA should be promoted, by whom, and/or at what intensity [11], as well as organisational barriers, including limited facilities and time [11].

\section{Aims}

The aim of the current study was to conduct an exploratory, large scale, international survey of pwCF, parents/caregivers of pwCF and HCP from CF multidisciplinary teams to explore attitudes and experiences of PA and PA promotion.

\section{Methods}

\section{Design}

The design was an online, descriptive, cross-sectional survey of pwCF, parents/caregivers of pwCF and HCP. All procedures performed in studies involving human participants were in accordance with the ethical standards of the institutional and/or national research committee and with the 1964 Helsinki declaration and its later amendments or comparable ethical standards. Ethical approval was obtained from the University of Exeter $(171025 / \mathrm{B} / 05)$ and all participants provided informed assent/consent prior to completing the survey.

\section{Participant sampling and data collection}

A convenience sample of people with $\mathrm{CF}$, parents/caregivers of pwCF and HCP in CF were recruited via social media or email. Participants were invited to complete a confidential online survey regarding their views, opinions and practices in relation to PA and PA promotion. A multifaceted approach to recruitment was employed. First, the invitation was shared through social media accounts, including the UK Cystic Fibrosis Trust, CF Ireland and the US CF Foundation. The survey link was also shared via the Cystic Fibrosis Foundation and Cystic Fibrosis Trust newsletters and email distribution lists. The survey was available in English, with no other exclusions or restrictions for participation. Data were collected between March 2018 and January 2019.

The survey was developed using Qualtrics software (Qualtrics, Provo, UT, USA) and comprised of three sets of questions: one each for pwCF, parents/caregivers of pwCF and HCP. On accessing the survey, participants were asked to identify their participant category and were then directed to the relevant participant information sheet. Survey questions were tailored to each participant group and included a combination of rating scales, multiple-choice questions and open-ended questions to capture views, experiences and practices relating to PA and PA promotion (online supplementary material 1).

All participants were asked to rate the importance of PA (both for themselves and for managing CF) using a visual-analogue scale (VAS) ranging from 1 "not important at all" to 10 "very important". Healthcare providers were then asked to complete a further 17 multiple-choice and open-ended questions relating to the differing roles and responsibilities for PA promotion within the clinical team, the nature of PA advice and recommendations given to pwCF, barriers to PA promotion, and their needs for improving PA promotion. pwCF and parents/caregivers of pwCF were asked to complete 20 multiple-choice and 
open-ended questions relating to their/their child's current PA behaviour and motivations, and any barriers and facilitators to being active. Finally, at the end of each section, all participants were given the opportunity to mention anything that had not been covered through free text fields.

\section{Data analysis}

Frequencies and descriptive statistics were used to analyse the quantitative data, using the statistical package for social science (SPSS version 25, IBM Corp., Armonk, NY, USA). Free text answers were analysed thematically [14] and used to offer further insight into, and explanations for, answers given to closed survey questions. All qualitative data were entered into the software NVivo 12 (QSR International Pty Ltd.).

\section{Results}

A total of 456 respondents started, and 351 respondents (105 HCP, 120 pwCF and 126 parents/caregivers) from 12 countries completed the survey. Participant demographics are shown in table 1.

\section{Importance of physical activity}

People with CF and their parents/caregivers rated the importance of PA highly for themselves personally (mean \pm SD $8 \pm 2$ and $8 \pm 1$, respectively), and in the management of their/their child's CF ( $9 \pm 1$ and $9 \pm 1$, respectively). Similarly, HCP rated the importance of PA in the management of CF highly $(10 \pm 1)$. However, when asked to rate the importance placed on PA from the perspective of other HCP in their teams, the mean \pm sD score was $7 \pm 1$.

\section{Motives for physical activity}

Across all countries, the most common motives for PA among pwCF included health (82\%) and enjoyment (49\%; table 2). Many participants' felt PA had a key role in their clinical management and maintaining their health (table 3; example 1). Some participants also perceived psychological benefits of PA that included enhanced confidence and control over their condition and enhanced sense of normality (table 3; example 2). While the health benefits of PA were recognised, this alone was not always sufficient motivation, particularly when the "reward" was not immediate (table 3; example 3).

\section{Physical activity behaviour}

Popular activities in which pwCF regularly (at least once a week) participate included: walking (85\%), running (35\%), going to the gym (37\%), weight-lifting (35\%), cycling (22\%), fitness classes (19\%), swimming (14\%), team sports $(9 \%)$ and yoga (6\%). The majority of respondents were physically activity when alone (74\%), whereas only $30 \%$ reported being active with friends (30\%), and with a family member $(29 \%)$ or spouse $(29 \%)$. There was considerable variation in reports of the type, duration, frequency and intensity of PA (table 3; example 4).

$48 \%$ of pwCF indicated that they would like to do more PA. The most common barriers to PA, regardless of location, were tiredness (61\%), lack of time (49\%) and illness (36\%) (table 4). Lack of enjoyment in PA was reported as a barrier by $21 \%$ of pwCF from the USA and $21 \%$ of pwCF from the UK. However, it was not reported as a barrier to PA by any individuals from other locations. Parents/caregivers of pwCF reported a lack of time for both themselves (33\%) and their child (36\%), tiredness (7\%), illness (29\%), lack of motivation (26\%) and school pressure (25\%) were the biggest barriers to their child being active.

pwCF highlighted the additional challenges they had to confront while being active with CF, including coping with the stigma and the demoralising cycle of building fitness with a chronic condition (table 3; example 5). However, for parents/caregivers of pwCF structural barriers appeared to be more of an issue. Specifically, cost of activities, lack of time and environmental barriers featured frequently (table 3; example 6). Many participants in the parents/caregivers category identified the role of the family: parents/ caregivers acting as role models or offering tangible support to help overcome barriers to PA for their child (table 3; example 7).

\section{Physical activity promotion}

The majority (90\%) of HCP reported discussing PA during every appointment, and $98 \%$ gave advice to patients about PA. Regardless of country discussions about PA were primarily considered the responsibility of the physiotherapist $(60 \%)$, and $75 \%$ of respondents reported that advice is based on one of the 19 guidelines (online supplementary material 2). Information largely focused on providing individualised information about the benefits of PA, and how to make it enjoyable and sustainable (table 3; example 8). 


\section{TABLE 1 Participant characteristics}

n $(\%)$

\section{Healthcare professionals ( $n=105$ )}

Location

Australia

$17(16)$

Canada

2 (2)

France

1 (1)

New Zealand

5 (5)

Switzerland

$1(1)$

The Netherlands

UK

40 (38)

USA

$22(20)$

Clinical population

Missing

8 (8)

Adult

35 (33)

Paediatric

27 (25)

Combined

$40(38)$

Position within the clinic

Missing

3 (3)

Doctor

$6(6)$

Nurse

$12(11)$

Dietician

1 (1)

Physiotherapist

$49(46)$

Manager

1 (1)

Technician

5 (5)

Administration

1 (1)

Physiologist

$4(4)$

Research

$4(4)$

Social worker

$2(2)$

Therapy practitioner

1 (1)

Other (not specified)

10 (9)

\section{Person with CF ( $n=120)$}

Location

Missing

$9(8)$

Australia

2 (1)

Canada

2 (1)

Germany

$1(1)$

India

$1(1)$

New Zealand 2 (1)

Spain

2 (1)

UK

USA

Age bracket

Missing

2 (1)

$\leqslant 12$ years

$1(1)$

13-18 years

4 (3)

19-35 years

$74(61)$

36-60 years

$37(30)$

$>60$ years

4 (3)

Sex

Male

41 (34)

Female

79 (66)

\section{Parents/caregivers of pwCF ( $n=126$ )}

Location

Australia

3 (2)

Bulgaria

India

$1(1)$

New Zealand

Spain

$1(1)$

Switzerland

UK

48 (37)

USA

$63(48)$

Age bracket of son/daughter with CF

$\leqslant 12$ years

44 (35)

13-18 years

19-35 years

Living with pwCF

Yes

Other

CF: cystic fibrosis; pwCF: people with cystic fibrosis. 
TABLE 2 Assessment of importance, confidence and enjoyment of physical activity (PA), and motives for PA among people with cystic fibrosis (CF) by location

\begin{tabular}{|c|c|c|c|c|c|c|c|}
\hline $\begin{array}{l}\text { Location } \\
\text { (n) }\end{array}$ & $\begin{array}{l}\text { Importance of } \\
\text { PA }\end{array}$ & $\begin{array}{c}\text { Importance of PA in the } \\
\text { management of CF }\end{array}$ & Confidence & Enjoyment & $\begin{array}{l}\text { Motive: } \\
\text { health }\end{array}$ & $\begin{array}{c}\text { Motive: } \\
\text { enjoyment }\end{array}$ & $\begin{array}{l}\text { Motive: time with } \\
\text { others }\end{array}$ \\
\hline USA (71) & $8 \pm 2$ & $9 \pm 1$ & $6 \pm 2$ & $8 \pm 2$ & $87 \%$ & $49 \%$ & $44 \%$ \\
\hline UK (37) & $8 \pm 2$ & $9 \pm 2$ & $6 \pm 2$ & $7 \pm 2$ & $73 \%$ & $48 \%$ & $23 \%$ \\
\hline Other (12) & $9 \pm 1$ & $9 \pm 1$ & $7 \pm 2$ & $9 \pm 1$ & $83 \%$ & $50 \%$ & $41 \%$ \\
\hline All (120) & $8 \pm 2$ & $9 \pm 1$ & $6 \pm 2$ & $8 \pm 2$ & $82 \%$ & $49 \%$ & $37 \%$ \\
\hline
\end{tabular}

Information was also provided on the composition of PA (type, frequency, duration and intensity); however, these recommendations were variable, and there did not appear to be any consistency within locations or age groups (table 3; example 9). Respondents reported that personalised PA or exercise programmes were available (70\%). However, the type of exercise programmes and PA promotion offered to pwCF varied based on access to formal exercise testing results and access to community services and staffing (table 3; example 10). HCP reported that more time, staff and training were required to maximise PA promotion, as well as more and improved exercise equipment and facilities and improved links to existing services within the community (e.g. cheaper gyms/reduced fees for community resources; table 3; example 11).

When asked about their clinical experience, 39\% of pwCF and 50\% of parents/caregivers reported that physiotherapists were most likely to talk about PA during all or most appointments. 9\% of pwCF and 7\% of parents/caregivers stated that none of the clinical team spoke about PA during all or most appointments. Common topics of conversation reported by pwCF included: current activity levels (75\%), changes to PA levels (40\%), ways of increasing PA (26\%), and information regarding duration (26\%) and types (37\%) of activities they should be doing (table 5).

Information regarding the intensity of PA (14\%) and addressing any specific concerns that pwCF may have $(5 \%)$ were less frequently mentioned. $17 \%$ of pwCF reported having concerns and questions about PA that had not been answered by the clinics; some participants wanted specific and personalised information for them/their child as individuals (table 3; example 12). pwCF and parents/caregivers did not consider discussions about PA to be sufficient, particularly for those who are sedentary (table 3; example 13). Facilities were also viewed as substandard (table 3; example 14).

\section{Discussion}

This study identified near unanimous agreement regarding the importance of PA for CF evidenced from both the qualitative and quantitative data collected from a wide international sample, although there remains considerable variation in PA practices and promotion for pwCF. In accordance with previous literature exploring the reasons for which PA is considered to be important for CF [11, 15], many pwCF attributed their health, and even their life, to their PA. Health and enjoyment were two of the biggest motivators for PA among pwCF, a finding that is consistent with motives for PA among both other clinical and healthy populations $[16,17]$. Those motivated by the physical and psychological health benefits of PA identified how PA helped make them feel in control, and restored a sense of normality; factors that have been linked to improvements in well-being [18], quality of life [19] and improved self-management practices [20].

Despite knowing that PA was important, motivation was an issue for some respondents. Knowledge of health benefits were not sufficient to make PA a priority, with individuals reporting that they were unable to fit PA into their day. Self-determination theory (SDT) describes two main reasons that people are not motivated: they are not interested in PA or its associated outcomes, or they do not feel sufficiently competent [21]. SDT posits that motivation may be due to the interplay between autonomy, competence and relatedness. People are more likely to engage in PA if it is their PA goal rather than it is imposed on them (autonomy), and more likely to be active when there is an opportunity for increased social interaction (relatedness) [22]. For pwCF who struggle with motivation for PA, it may be beneficial to emphasise the range of activities (i.e. not just exercise) and benefits associated with PA (i.e. improved mental and social well-being, increased enjoyment associated with specific activities). Participants in the present study identified a wide range of PA behaviours that are in keeping with activities chosen by their (non-CF) peers $[23,24]$. Tailoring suggestions for PA to individual motives for PA (e.g. recommending pleasurable or social activities for those who are not motivated by health) may be beneficial for promoting activity [25]. 
TABLE 3 Participant quotes

Example 1 "It is the reason I am still alive. Consistent exercise has been a life saver. One of the most important parts of my treatment plan" (Rebecca, pwCF, 36-60 years, USA).

Example 2 "It makes me feel like I can beat CF" (Chloe, pwCF, 19-35 years, USA).

"If we do exercise daily then we are none other than normal people" (Paul, pwCF, 19-35 years, UK).

"As a youngster I liked doing exercise because it was not 'medical' but something I could do that I enjoyed, helped me feel normal and bond with peers and I knew it was good for me" (Daniel, pwCF, 19-35 years, New Zealand).

Example 3 "As much as I logically understand how important it is, it's not always enough to get me moving physically" (Claire, pwCF, 19-35 years, USA).

"There isn't a big enough reward to be more active yet. She is at a good weight and feels healthy. Doing something else with her time is more rewarding than exercising" (Erica, parent/caregiver, UK).

"I know logically I need to help my child be healthy, but I just don't know the best way to keep us both motivated" (Tina, parent/ caregiver, USA).

Example 4 "I weed my garden and walk my dog 5 days a week about a half mile" ( Jessica, pwCF, 36-60 years, UK).

"I train in a gym every day. I take one rest day in every 10 or so" (Mark, pwCF, 19-35 years, UK).

Example 5 "It is hard sometimes when people are staring at me in [fitness] class because I cough a lot" (Kate, pwCF, 13-18 years, UK).

"When you become less well (e.g. an infection or just a blip) it's a downwards spiral: you don't have the energy to do the exercise when you need it most, and as you are not exercising then restarting becomes more difficult. With CF being so unstable this constant cycle of working hard to just get unwell and lose your gains and have to start all over again - that's tough to keep picking yourself up again to start from the beginning all over" (Isabelle, pwCF, 19-35 years, UK).

Example 6 "My daughter loves gymnastics and soccer, but we can't afford the cost" ( Justine, parent/caregiver, USA).

"We fear group activities with mixing he could get ill" (Adam, parent/caregiver, UK).

Example 7 "We had to explain to school that exercise is at times going to be prioritised over homework in order to keep her well" (Louise, parent/caregiver, UK).

"I fully believe my son would not be here if I hadn't started to run with him years ago" ( Jason, parent/caregiver, USA)

Enjoyment. It can be seen as a chore, something they HAVE to do, the difficulty is keeping it enjoyable (Karen, parent/caregiver, UK).

Example 8 "[Information is] based on exercise test results, disease severity and patient preference" ( Jennifer, HCP, UK, combined).

" $[\mathrm{We}]$ help them to explore and find something that they like doing, find something with friends to make it social, how to fit into their already busy schedule" (Grace, HCP, Australia, paediatric).

Example 9 "We advise a mixture of cardio and strength training" (Isla, HCP, UK, adult)

"Aiming 60 min of activity/exercise daily" (Charlie, HCP, UK, paediatric).

"Aiming for minimum of 30 min of moderate- to high-intensity of physical activity five times a week" (George, HCP, Australia, paediatric).

"Aim to exercise (20-30 mins per day) 5 days a week. Also "micro" episodes of exercise are also good for you (e.g. taking stairs rather than lift, parking an extra block away and walking, etc.)" (Mia, HCP, New Zealand, combined).

Example 10 "[We provide] gym with many activities for in- and out-patients le.g. table tennis swing ball, traditional gym equipment, weight training, etc.). Dance and Wii fit activities in in-patient rooms. Yoga and personal training with personal trainers via video link. Treasure hunt around the hospital. Community visits from personal trainer with specific plan. Support to start using community facilities in patient's location and including accessing finance to support this where necessary. Emphasis on patient choice and lifestyle. Bikes and Xbox for exercise in all in-patient rooms" (Oscar, HCP, UK, adult).

"[PA is] currently not part of standard care" (Lily, HCP, Canada, paediatric).

Example 11 "[We need] dedicated exercise time and skills - preferably by an exercise physiologist" (Helen, HCP, UK, paediatric)

"More support from local gyms offering training packages at reduced rates... Some children don't like gyms so maybe more support from leisure centres to offer help with other children's classes - dance/roller skating/trampolining etc." ( John, HCP, UK, adult).

Example 12 "Where do I begin? Ha!" (Marie, pwCF, 36-60 years, USA)

"I would like to know more about specific exercises that are most beneficial to me" ( Jane, pwCF, 19-35 years, USA)

"I want to know what I can do with my child to help them live longer. "Be active" is not a satisfactory answer anymore" (Emily, parent/caregiver, UK).

Example 13 'It's not discussed nearly enough. It's just a 'are you exercising' and that's it" (Amy, pwCF, 36-60 years, USA)

"Clinic is rushed. Exercise is only mentioned if we bring the subject up. All physios want is a quick cough swabs and FEV ${ }_{1}$ and FVC results" (Ryan, parent/caregiver, UK).

"Needs more specialised advice for sedentary parents, saying your child needs to be active isn't enough as neither of us have exercised since primary school" (Gemma, parent/caregiver, UK).

Example 14 "Our hospital has no way to exercise in-patients. No exercise room, no playground. Rules keep us inside our room. It's terrible. If exercise is important, our team and physical environment at the hospital needs to support that" (James, parent/caregiver, USA).

Quotes are followed by pseudonyms, whether the respondent is a pwCF, HCP or parent/caregiver, age bracket, and location. Quotes from HCP are followed by "paediatric" "adult" or "combined" to indicate the type of clinic population. CF: cystic fibrosis; pwCF: people with cystic fibrosis; HCP: healthcare professionals; $\mathrm{FEV}_{1}$ : forced expiratory volume in $1 \mathrm{~s}$; FVC: forced vital capacity. 
TABLE 4 Barriers to physical activity (PA) reported by people with cystic fibrosis (pwCF) and parents/caregivers of pwCF by location

\begin{tabular}{|c|c|c|c|c|c|c|c|c|c|c|}
\hline Subjects $\mathbf{n}$ & $\begin{array}{c}\text { Time } \\
\text { (parent) }\end{array}$ & $\begin{array}{c}\text { Time } \\
\text { (self/child) }\end{array}$ & Tired Illness & $\begin{array}{c}\text { Lack of } \\
\text { enjoyment }\end{array}$ & $\begin{array}{c}\text { Low } \\
\text { motivation }\end{array}$ & $\begin{array}{l}\text { School } \\
\text { work }\end{array}$ & $\begin{array}{l}\text { Concern } \\
\text { about } \\
\text { symptoms }\end{array}$ & $\begin{array}{c}\text { Concern } \\
\text { about } \\
\text { weight loss }\end{array}$ & $\begin{array}{l}\text { Concern } \\
\text { about PA } \\
\text { with CF }\end{array}$ & $\begin{array}{c}\text { Concerns } \\
\text { about type/ } \\
\text { intensity of PA }\end{array}$ \\
\hline
\end{tabular}

\begin{tabular}{|c|c|c|c|c|c|c|c|c|c|c|c|c|}
\hline \multicolumn{13}{|l|}{ pwCF } \\
\hline USA & 71 & NA & $50 \%$ & $67 \%$ & $46 \%$ & $21 \%$ & $33 \%$ & $8 \%$ & $16 \%$ & $8 \%$ & $8 \%$ & $9 \%$ \\
\hline UK & 37 & NA & $48 \%$ & $54 \%$ & $24 \%$ & $21 \%$ & $24 \%$ & $0 \%$ & $8 \%$ & $10 \%$ & $0 \%$ & $8 \%$ \\
\hline Other & 12 & NA & $41 \%$ & $50 \%$ & $16 \%$ & $0 \%$ & $8 \%$ & $16 \%$ & $0 \%$ & $0 \%$ & $16 \%$ & $8 \%$ \\
\hline \multicolumn{13}{|c|}{ Parents/caregivers } \\
\hline USA & 63 & $38 \%$ & $28 \%$ & $4 \%$ & $25 \%$ & $17 \%$ & $27 \%$ & $22 \%$ & $3 \%$ & $7 \%$ & $4 \%$ & $1 \%$ \\
\hline UK & 48 & $33 \%$ & $29 \%$ & $8 \%$ & $33 \%$ & $23 \%$ & $29 \%$ & $29 \%$ & $2 \%$ & $6 \%$ & $4 \%$ & $4 \%$ \\
\hline
\end{tabular}

In agreement with previous research $[12,13,26]$, barriers to PA reported by parents/caregivers and pwCF were tiredness and time, along with structural barriers (cost, environment). Previous research found that parental stress, tiredness and value placed on PA influences the context in which PA occurs and may reduce overall levels of PA among pwCF [20]. As parents play a critical role in promoting PA for young people [27], it is crucial that HCP consider barriers identified by both parents/caregivers and pwCF. Within the current study, and in line with previous qualitative research [28], parents often acted as enablers or role models to support their children to be active. For parents identifying as sedentary, advice to be active for and with their child could be overwhelming and additional support for these parents/ families could be required. Promoting PA as a lifestyle choice for the whole family, rather than a treatment for the pwCF, may be an effective way of enhancing engagement with PA [29].

Whilst HCP considered PA to be important, they perceived that their colleagues do not rate PA as highly. This is potentially due to the high percentage of HCP respondents being physiotherapists, a role that many participants ( $\mathrm{pwCF}$ and $\mathrm{HCP}$ ) claimed to be predominantly responsible for discussions about PA during clinics. However, messages relating to the importance of PA are likely to be stronger and more effective if they are disseminated by multiple and trusted sources [30]. If trusted members of the clinical team do not value PA or consider PA promotion to solely be the role of the physiotherapist, messages relating to the importance of PA may be weakened. Indeed, parents/caregivers highlighted the discordance between messages relating to the importance of PA, and hospital settings in which PA was not viable. Education for members of clinical teams who do not view PA as highly, or do not consider PA promotion to be their role, must ensure that messages relating to the importance of PA are consistent.

There also appears to be a disconnect between HCP reports of discussing PA ( $90 \%$ for all or most clinic appointments) compared with pwCF interpretation of the frequency of discussion. This discrepancy could be explained by the majority of HCP in this sample being from the UK, whereas the majority of pwCF were not. Also, the HCP approach for providing advice on how to make PA fun, enjoyable and sustainable may not match the specific concerns of pwCF regarding optimal activity type, frequency, intensity and duration, particularly for those who are already active. Congruent with previous research [11], a lack of time, staff and training were highlighted by HCP as the biggest barrier to PA promotion. While some HCP described well-equipped facilities, this was not always the case. Many pwCF and parents/caregivers

TABLE 5 Discussions relating to physical activity (PA) reported by people with cystic fibrosis by location

\begin{tabular}{|c|c|c|c|c|c|c|c|c|}
\hline & $\begin{array}{c}\text { Subjects } \\
n\end{array}$ & $\begin{array}{c}\text { Current PA } \\
\text { levels }\end{array}$ & $\begin{array}{c}\text { Changes in } \\
\text { PA }\end{array}$ & $\begin{array}{c}\text { Ways of } \\
\text { increasing PA }\end{array}$ & $\begin{array}{c}\text { Duration of } \\
\text { PA }\end{array}$ & $\begin{array}{c}\text { Types of } \\
\text { PA }\end{array}$ & $\begin{array}{c}\text { Intensity of } \\
\text { PA }\end{array}$ & $\begin{array}{c}\text { Specific } \\
\text { concerns }\end{array}$ \\
\hline USA & 71 & $73 \%$ & $46 \%$ & $21 \%$ & $28 \%$ & $38 \%$ & $12 \%$ & $2 \%$ \\
\hline UK & 37 & $83 \%$ & $35 \%$ & $37 \%$ & $24 \%$ & $40 \%$ & $16 \%$ & $8 \%$ \\
\hline Other & 12 & $58 \%$ & $25 \%$ & $25 \%$ & $25 \%$ & $25 \%$ & $16 \%$ & $16 \%$ \\
\hline All & 120 & $75 \%$ & $40 \%$ & $26 \%$ & $26 \%$ & $37 \%$ & $14 \%$ & $5 \%$ \\
\hline
\end{tabular}


described poor facilities available, rushed clinic appointments and underplay the role of PA (particularly for the more motivated).

\section{Implications for policy and practice}

The current findings highlight substantial differences in the facilities, staffing and training, and time available to dedicate to PA between clinics. HCP reported use of 19 different guidelines, resulting in differences in recommendations provided to pwCF. Training HCP in the use of an agreed set of guidelines would ensure that advice given to patients is consistent. Such a document may take the form of a consensus document, in which existing guidelines are synthesised and agreed among experts.

Our research also identified a range of motives, barriers and facilitators to PA among pwCF. Variation in the motives underpinning PA, as well as substantial differences in the types, frequencies, durations and intensities of PA behaviour, suggest the need for a targeted approach to PA promotion. A clinical tool to identify which patients may benefit from each form of support may also be useful.

\section{Strengths and limitations of the study}

To our knowledge, this is the first study to simultaneously explore attitudes and experiences of PA among pwCF, parents/caregivers of pwCF and HCP and presents the most comprehensive overview of PA and PA promotion in multiple countries. The integration of both qualitative and quantitative methods enabled us to obtain a greater insight into the views and experiences of participants than traditional closed questions or interviews, which are often used in isolation.

A major limitation of this work relates to the generalisability of findings to a wider population. Whilst every attempt was made to recruit participants from a range of age groups and countries, the majority of our participants were recruited through two large organisations located in the UK (The Cystic Fibrosis Trust) and the USA (Cystic Fibrosis Foundation). This resulted in a sample overly represented by participants from the UK and the USA, with the majority (92\%) between the ages of 19 and 60 years of age. Furthermore, due to the nature of the survey, it is possible that the sample is biased in favour of those with a particular interest in PA. This is further evidenced by the fact that nearly half of the HCP were physiotherapists who may prioritise PA over other members of the CF team. In addition, no information was collected on the health status or lung function of the participants with CF. Lung function is a known determinant of participation in PA [1-3]; and therefore, there is the potential that the sample is biased in favour of those who are relatively well and/or have a mild form of CF. Generalisations from this work beyond this population must be made with caution.

This survey was designed to be exploratory in nature, and to capture the views of a range of individuals with CF and their support teams. Our intention was not to estimate population means, or to statistically compare the three groups of participants. As a result, we did not set out to recruit a specific number of participants in each group of interest to enable such analyses to be conducted. With data generated through this survey, we hoped to equip HCP with the knowledge pertaining to the types of activities performed by pwCF, barriers and facilitators to PA, and perceptions of PA promotion within clinics. We hope that this will allow HCP to consider the issues identified within the manuscript when attempting to promote PA among pwCF.

\section{Conclusions}

This research is the first mixed methods survey to present a thorough description of PA and PA promotion from the perspective of pwCF and their support teams around the world. Despite the majority of participants rating the importance of PA highly, substantial variability exists regarding the facilities and clinical support available to them, as well as why and how people are active. This research highlights the need for the identification of what constitutes "best practice" in relation to PA promotion within clinics, as well as an increased awareness of the different roles PA can play in the lives of pwCF. Such understanding is critical if clinical teams are to provide support and advice that is tailored to individual motives and circumstances.

Acknowledgements: We would like to thank the Cystic Fibrosis Trust for funding the research project, and for their support with recruitment of participants. We would also like to thank the Cystic Fibrosis Foundation for their help with recruitment of participants. Finally, we would also like to thank the participants for their valuable time in participating in this study.

Data availability: All available data can be obtained by contacting the corresponding author.

Conflict of interest: S. Denford reports grants from Cystic Fibrosis Trust during the conduct of the study. N.S. Cox has nothing to disclose. K.A. Mackintosh has nothing to disclose. M.A. McNarry has nothing to disclose. P. O'Halloran has nothing to disclose. A.E. Holland has nothing to disclose. O.W. Tomlinson has nothing to disclose. A.R. Barker reports 
grants from Cystic Fibrosis Trust Strategic Research Centre award during the conduct of the study. C.A. Williams reports grants from Cystic Fibrosis Trust during the conduct of the study.

Support statement: This work was funded by the Cystic Fibrosis Trust Strategic Research Centre grant number 008 . Funding information for this article has been deposited with the Crossref Funder Registry.

\section{References}

1 Williams CA, Benden C, Stevens D, et al. Exercise training in children and adolescents with cystic fibrosis: theory into practice. Int J Pediatr 2010; 2010: 670640.

2 Williams CA, Stevens D. Physical activity and exercise training in young people with cystic fibrosis: Current recommendations and evidence. J Sport Health Sci 2013; 2: 39-46.

3 Cystic Fibrosis Trust. Standards of care and good clinical practice for the physiotherapy management of cystic fibrosis (Third edition). www.cysticfibrosis.org.uk/the-work-we-do/resources-for-cf-professionals/consensusdocuments. Date last updated: 2017. Date last accessed: 4 May, 2020.

4 Cystic Fibrosis Foundation. Why fitness matters. www.cff.org/Life-With-CF/Daily-Life/Fitness-and-Nutrition/ Fitness/Why-Fitness-Matters/. Date last accessed: 4 May, 2020.

5 Selvadurai HC, Blimkie C, Cooper P, et al. Gender differences in habitual activity in children with cystic fibrosis. Arch Dis Child 2004; 89: 928-933.

6 Mackintosh KA, Ridgers ND, Evans RE, et al. Physical activity and sedentary time patterns in children and adolescents with cystic fibrosis and age- and sex-matched healthy controls. J Phys Act Health 2018; 15: 82-88.

7 Guthold R, Stevens GA, Riley LM, et al. Worldwide trends in insufficient physical activity from 2001 to 2016: a pooled analysis of 358 population-based surveys with 1.9 millions participants. Lancet Glob Health 2018; 6 : e1077-e1086.

8 Rowbotham N, Smith S, Leighton PA, et al. Question CF: a James Lind Alliance Priority Setting Partnership in cystic fibrosis. J Cyst Fibros 2017; 16: Suppl. 1, S38.

9 Fisher J, Fisher W. Changing AIDS-risk behaviour. Psychol Bull 1992; 111: 455-457.

10 Sallis J, Owen N. Ecological models of health behaviour. In: Glanz K, Rimmer B, Lewis F, eds. Health Behavior and Health Education. San Francisco, Jossey-Bass: San Francisco, 2007; pp. 462-484.

11 Denford S, MackIntosh KA, McNarry MA, et al. Promotion of physical activity for adolescents with cystic fibrosis: A qualitative study of UK multi-disciplinary cystic fibrosis teams. Physiotherapy 2020; 106: 111-118.

12 Moola FJ, Faulkner GE, Schneiderman JE. "No time to play": perceptions toward physical activity in youth with cystic fibrosis. Adapt Phys Activ Q 2012; 29: 44-62.

13 Shelley J, Fairclough SJ, Southern KW, et al. A formative study exploring perceptions of physical activity monitoring among children and young people with cystic fibrosis and health care professionals. BMC Pediatr 2018; 18: 335.

14 Braun V, Clarke V. What can "thematic analysis" offer health and wellbeing researchers? Int J Qual Stud Health Well-being 2014; 9: 26152.

15 Denford S, Hill DM, Mackintosh KA, et al. Using photo-elicitation to explore perceptions of physical activity among young people with cystic fibrosis. BMC Pulm Med 2019; 19: 220.

16 Kosteli M, Heneghan NR, Roskell C, et al. Barriers and enablers of physical activity engagement for patients with COPD in primary care. Int J Chron Obstruct Pulmon Dis 2017; 12: 1019-1031.

17 Roberts S, Reeves M, Ryrie A. The influence of physical activity, sport and exercise motives among UK based university students. J Furth High Educ 2015; 39: 598-607.

18 Moola FJ, Faulkner GE. 'A tale of two cases:' the health, illness, and physical activity stories of two children living with cystic fibrosis. Clin Child Psychol Psychiatry 2014; 19: 24-42.

19 Sawicki G, Sellers DE, Robinson WM. Associations between illness perceptions and health-related quality of life in adults with cystic fibrosis. J Psychosom Res 2011; 70: 161-167.

20 Moola FJ, Faulkner GEJ, Kirsh JA, et al. Developing physical activity interventions for youth with cystic fibrosis and congenital heart disease: Learning from their parents. Psychol Sport Exerc 2011; 12: 599-608.

21 Deci EL, Ryan RM. Intrinsic motivation and self-determination. In: Deci EL, Ryan RM, eds. Human behavior Perspectives in social psychology. New York, Plenum, 1985; p. 371.

22 Teixeira PJ, Carraca EV, Markland D, et al. Exercise, physical activity, and self-determination theory. Int J Behav Nutr Phys Act 2012; 9: 78.

23 Joint Health Surveys Unit. Health Surveys for England 2012: Physical activity and fitness. http://doc.ukdataservice. ac.uk/doc/7480/mrdoc/pdf/7480userguide.pdf. Date last updated: 2013. Date last accessed: May 2020.

24 Kjønniksen L, Torsheim T, Wold B. Tracking of leisure time physical activity during adolescence and young adulthood: a 10-year longitudinal study. Int J Behav Nutr Phys Act 2008; 5: 69.

25 Segar ML, Richardson CR. Prescribing pleasure and meaning: cultivating walking motivation and maintenance. Am J Prev Med 2014; 47: 838-841.

26 Swisher A, Erickson M. Perceptions of physical activity in a group of adolescents with cystic fibrosis. Cardiopulm Phys Ther J 2008; 19: 107-113.

27 Brown H, Atkin AJ, Pnater J, et al. Family based interventions to increase physical activity in children: a systematic review, meta-analysis and realist synthesis. Obes Rev 2016; 17: 345-360.

28 Fereday J, MacDougall M, Spizzo M, et al. “There's nothing I can't do-I just put my mind to anything and I can do it": a qualitative analysis of how children with chronic disease and their parents account for and manage physical activity. BMC Pediatric 2009; $1: 1$.

29 Segar ML, Guerin E, Phillips E, et al. From a vital sign to vitality: selling exercise so patients want to buy it. Transl J Am Coll Sports Med 2016; 1: 97-102.

30 Abraham C, Kools M. Writing Health Communication: An evidence based guide. London, SAGE Publications Ltd, 2011. 